\title{
BMJ Open Systematic literature review on which maternal alcohol behaviours are related to fetal alcohol spectrum disorders (FASD)
}

\author{
Sylvia Roozen, ${ }^{1,2}$ Gjalt-Jorn Ygram Peters, ${ }^{2,3}$ Gerjo Kok, ${ }^{1,2}$ David Townend, ${ }^{1,4}$ \\ Jan Nijhuis, ${ }^{1,5}$ Ger Koek, ${ }^{1,6}$ Leopold Curfs ${ }^{1}$
}

To cite: Roozen S, Peters G-JY, Kok G, et al. Systematic literature review on which maternal alcohol behaviours are related to fetal alcohol spectrum disorders (FASD). BMJ Open 2018;8:e22578. doi:10.1136/ bmjopen-2018-022578

- Prepublication history for this paper is available online. To view these files please visit the journal online (http://dx.doi org/10.1136/bmjopen-2018022578).

Received 13 March 2018 Revised 20 November 2018 Accepted 26 November 2018

\section{Check for updates}

(C) Author(s) (or their employer(s)) 2018. Re-use permitted under CC BY-NC. No commercial re-use. See rights and permissions. Published by BMJ.

For numbered affiliations see end of article.

\section{Correspondence to}

Sylvia Roozen;

sylvia.roozen@

maastrichtuniversity.nl

\section{ABSTRACT}

Objectives Fetal alcohol spectrum disorders (FASD) is a worldwide problem. Maternal alcohol consumption is an important risk factor for FASD. It remains unknown which alcohol consumption patterns most strongly predict FASD. The objective of this study was to identify these.

Design Systematic literature review.

Methods We searched in PubMed, PsychINF0, PsycARTICLES, ERIC, CINAHL, Embase and MEDLINE up to August 2018. The query consisted of keywords and their synonyms related to FASD, pregnancy and behaviour. Studies were excluded when not published in English, were reviews or involved non-human subjects. Substantial heterogeneity precluded aggregation or meta-analysis of the data. Instead, data were qualitatively inspected.

Results In total, 21 studies were eligible for further data analysis. All studies that measured both maternal alcohol drinking behaviours and FASD reported retrospective data on maternal drinking patterns, employing both continuous and categorical measures and exhibiting substantial heterogeneity in measures of alcohol consumption (eg, timing of exposure, quantification of alcohol measure and definition of a standard drink). Study quality improved over time and appeared higher for studies based on active case ascertainment, especially when conducted in schools and when behaviour was assessed through interviews.

Conclusions We aimed to identify specific maternal drinking behaviour(s) related to FASD. The state of the literature precludes such conclusions. Evidencebased preventive measures necessitate identifying which prenatal alcohol drinking behaviour(s) are most in need of intervention. Therefore, we formulate three recommendations for future research. First, future studies can optimise the value of the collected dataset through specifying measurements and reporting of maternal drinking behaviours and avoiding categorised measures (nominal or ordinal) whenever possible. Second, samples should not be selected based on FASD status, but instead, FASD status as well as maternal alcohol consumption should both be measured in a general population sample. Finally, we provide 10 reporting guidelines for FASD research.
Strengths and limitations of this study

- This systematic literature review uses a comprehensive search strategy to cover the published literature.

- We did not consult grey literature.

- Consultation about data aggregation took place with three independent alcohol experts.

- Substantial heterogeneity prevented synthesis but yielded a rich set of recommendations as to reporting guidelines and measurement principles.

\section{INTRODUCTION}

Prenatal alcohol exposure is one of the leading causes of mental retardation resulting in irreversible lifelong consequences for the unborn child (eg, neurocognitive deficits, growth deficiencies and facial dysmorphology). ${ }^{1}$ These adverse outcomes are also known as fetal alcohol spectrum disorders (FASD). The spectrum encompasses various diagnostic subtypes: fetal alcohol syndrome (FAS), partial fetal alcohol syndrome (pFAS), alcohol-related neurodevelopmental disorder (ARND), alcohol-related birth defects (ARBD) and neurobehavioural disorder with prenatal alcohol exposure (ND-PAE). ${ }^{12}$ Epidemiological research implies that FASD is a worldwide problem. Initial FAS prevalence estimates ranged from 0.5 to 7 per 1000 live births. ${ }^{3}{ }^{4}$ Recent systematic literature reviews ${ }^{5}{ }^{6}$ including multiple meta-analyses reported estimates ranging from 0.11 to 55.42 per 1000 (FAS), from 0.8 to 43.01 per 1000 (pFAS), from 0.12 to 20.25 per 1000 (ARND), from 1.03 to 10.82 per 1000 (ARBD) and from 1.06 to 113.22 per 1000 (FASD).

FASD, as its name implies, is caused by alcohol use. Several reviews have aimed to further elucidate the relationship between alcohol use and filial FASD. ${ }^{78}$ Specifically, mothers of children diagnosed in the FASD 
spectrum reported drinking levels ranging from mild to excessive ('binge drinking') alcohol use. ${ }^{7-13}$ The severity of FASD may be dependent on the level, pattern and timing of prenatal alcohol exposure before and during pregnancy, ${ }^{13} 14$ along with other confounding factors such as nutritional status of the mother (eg, vitamin or mineral intake), environmental factors (eg, social relationships, stress), maternal age and genetic makeup. ${ }^{14-16}$ As yet, there is no known safe amount of alcohol to drink while pregnant. $^{1131718}$

Two systematic literature reviews reported associations between level of alcohol exposure and negative effects on child development. ${ }^{711}$ Both reviews show the negative effects of higher amounts of alcohol intake (daily alcohol consumption up to four or more drinks per occasion before and during pregnancy) related to various neuropsychological outcomes (including but not specific for a FASD diagnosis). However, these reviews are inconclusive about behaviours related to the outcome of FASD specifically, ${ }^{5711}$ or the effects of consumption of lower amounts of alcohol.

Planning evidence-based health-promoting programmes requires an adequate understanding of which maternal behaviour(s) are associated with FASD. Note that maternal alcohol consumption is not the only factor for filial FASD. Paternal and even grandparental consumption patterns have also been implicated, ${ }^{19} 20$ but as yet it remains undecided whether paternal and grandparental consumption should also be included in the FASD definition (effects of paternal and grandparental consumption are considered necessarily either genetic or through influencing maternal alcohol consumption, whereas maternal alcohol consumption has a direct teratogenic effect). However, for the sake of this review, we limited ourselves to maternal alcohol consumption. Specifically, a first step for designing prevention programmes requires defining specific target behaviour(s) of the target population related to FASD. ${ }^{61}$ However, the literature remains inconclusive about which maternal drinking behaviours are related to alterations of the fetal development. Despite this conflicting and inconclusive evidence of the negative effects on the developing fetus, public health recommendations are made nonetheless. These recommendations share one common principle, namely that complete abstinence of alcohol use during pregnancy is the safest approach to prevent any possible risks to the unborn child. ${ }^{1131718}$ However, despite this common thread, there are also many differences between the recommendations. For example, the British Medical Association lists four different recommendations that are currently made in the UK alone..$^{13}$ This heterogeneity is problematic because communicating multiple contrasting recommendations is confusing for the target audiences. At the same time, there are good arguments to tailor the recommendations. For example, it is likely that although any alcohol consumption may entail risks, binge drinking (BAC to $0.08 \mathrm{~g}$ per cent or above; four or more drinks in about 2 hours) is one of the serious risk factors and associated with severe forms of FASD. ${ }^{22}$ Therefore, it appears that special attention for specific risk groups such as heavily drinking pregnant women is warranted.

Yet, implementing such a tailored approach is currently hindered by the lack of knowledge regarding the doseresponse relationship and potential moderators. On the one hand, insufficient evidence is available about the association of different alcohol-related behaviours to FASD-related risk, especially low doses of alcohol, to adequately delineate target groups to enable tailored communication. This would seem to justify foregoing the heterogeneous recommendations and instead converging on an abstinence recommendation. However, in some target populations, such a total abstinence recommendation does not seem feasible. Especially high-risk populations, for example, heavily drinking women, may not be able to completely eliminate their alcohol intake, for example, because of personal factors as self-regulation skills, or environmental factors such as social pressures. Given that a total abstinence recommendation may be unrealistic for some of the highest risk populations, such a recommendation can be ethically problematic.

To illustrate this, consider figure 1 . This figure shows two potential dose-response relationships between weekly maternal alcohol consumption and risk of filial FASD for a given individual (note that individual vulnerabilities can vary). The left panel shows a sigmoid relationship, where risk remains low if less than five units are consumed weekly, whereas in the linear dose-response relationship depicted in the right panel, risk is already considerable at five consumptions weekly. For those subpopulations where abstinence recommendations may be unrealistic, if the dose-response relationship is similar to that shown in the left panel, a harm reduction message such as "consume at most five units' (the yellow areas in figure 1) may be easier to defend than if the dose-response relationship is linear. Not only may such a message be easier to defend, it may be more effective at decreasing FASD prevalence. Setting unachievable goals has little behaviour change potential, ${ }^{23}$ and if a more achievable goal can stimulate the target population to moderate their alcohol intake enough to decrease the risk of FASD, while an abstinence message, being unrealistic, has no effect, the ethics of an abstinence message become questionable. If, however, the risk increases very rapidly even with light alcohol consumption, deviating from an abstinence message may be damaging.

Animal models have provided some evidence as to potential dose-response relationships. However, such models are not fully translatable to humans, ${ }^{16}$ and especially given that the present research question concerns not simply whether a dose-response relationship exists, but what the nature of this relationship is, relying on animal models does not seem appropriate.

Further research is warranted to identify behaviours for health promotion programmes to target on. Developing health promoting programmes aiming at reducing alcohol consumption during pregnancy first requires 
Potential dose-response relationships: sigmoid

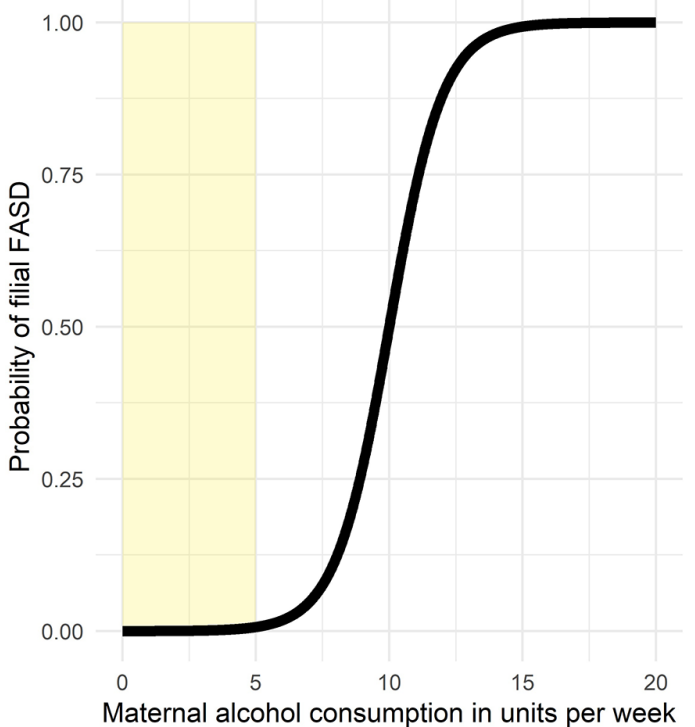

Potential dose-response relationships: linear

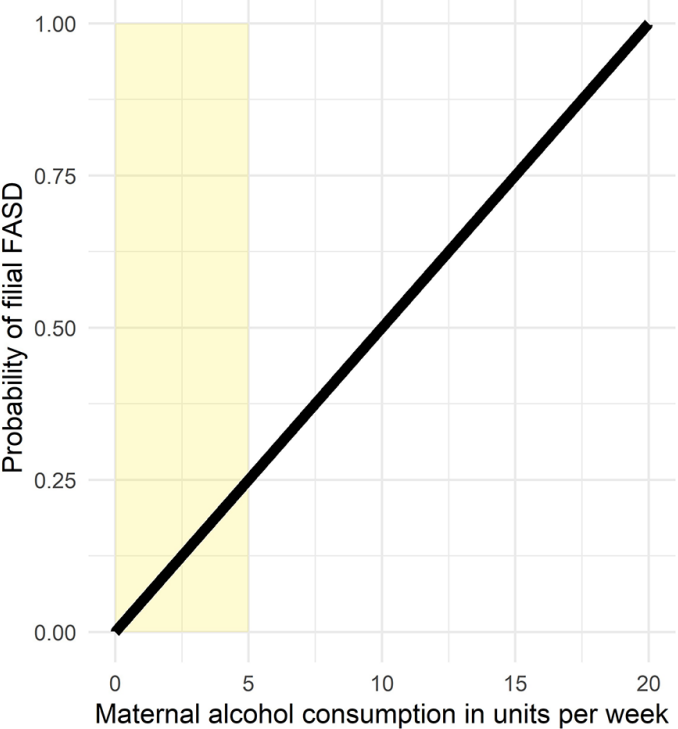

Figure 1 Two examples of possible dose-response relationships between maternal alcohol consumption and probability of filial FASD. FASD, fetal alcohol spectrum disorders.

identifying which prenatal alcohol drinking behaviour(s) are most in need of intervention. The purpose of the present study is to conduct a systematic literature review and meta-analysis to identify those maternal alcohol drinking behaviours most strongly related to FASD.

\section{MATERIALS AND METHODS}

Protocol and data repository

Data will be reported following the Preferred Reporting Items for Systematic Reviews and Meta-Analyses guideline. ${ }^{24}$ All materials and supporting documents are publicly available at the Open Science Framework repository at https://osf.io/whq45/. In this repository, we have numbered the directories that organise the materials. Hereafter, we will refer to materials in this repository as 'resource 1' through 'resource 8', which correspond to these directories.

\section{Ethics statement and patient and public involvement}

The current study extracted data from online databases and did not involve participation of participants; therefore, it was not necessary to obtain ethical permission.

\section{Search strategy}

A search was conducted in PubMed, PsychINFO, PsychARTICLES, ERIC, CINAHL, Embase and MEDLINE databases up to August 2015 using an extensive query consisting of keywords related to FASD, pregnancy and behaviour (eg, FAS, pregnancy, alcohol use and risk factor). We reran the query just before submitting the manuscript in August 2018 and performed a cursory inspection to scan for newly added papers. Moreover, we applied the ascendancy approach by inspecting the reference lists of included articles (the complete queries are included in resource 1).

\section{Study selection}

Resulting hits from the query were exported and screened by two independent screeners in three rounds. The first screening round was based on titles only; the second, on titles and abstracts; and the third, on the full-text articles. Records were included if they were written in English and reported maternal alcohol-related behaviours associated with a FASD diagnosis. Records that were duplicates, concerned reviews or meta-analysis, or concerned studies that involved non-human subjects were excluded. An extensive list of inclusion and exclusion criteria is located in the screening instructions (resource 2).

\section{Data extraction}

Data were transferred onto extraction forms, which were templated source code files for $\mathrm{R}^{25}$ using Notepad++. Researcher SR completed all extraction forms including the following variables: sampling method (retrospective vs prospective), sampling selection (select vs aselect), variables on which controls were matched (eg, age mother and study year of the child), recruitment setting (eg, school and clinic), descent (native vs non-native population), geography, year of data collection, sample size, subsamples, method of diagnosis (eg, Institute of Medicine (IOM) and 4-digit), syndrome category (eg, FAS and ARND), datatype (eg, aggregate and question), datatype levels (eg, nominal and logical), confirmed maternal alcohol exposure, method of case ascertainment (active vs passive) and data collection method (self-report vs interview). Moreover, variables related to drinking behaviours were extracted. Specifically, period of alcohol 
consumption (eg, first trimester and before pregnancy), timeframe (concurrent vs retrospective), intensity specification (eg, any day and weekend day), specification of units (eg, oz and $\mathrm{mg}$ ), specification of timeframe (eg, per year and per month), bingeing and alcoholism. Also, when no indication of one standard drink was provided, the units in grams were granted depending on country and their national alcohol guidelines (eg, one standard drink in the $\mathrm{USA}=14 \mathrm{~g}$, Australia $=10 \mathrm{~g}$; see resource $5)$. These extraction forms were then read into $R$ and processed by an R script.

\section{Quality assessment}

A slightly adapted version of the Newcastle-Ottawa Scale (NOS) was used for assessing the quality of non-randomised studies for further meta-analysis with a maximum of $10 \operatorname{stars}^{26}$ (see resource 4 for the complete assessment and comparison with the original version). The quality of each publication was assessed by two independent reviewers (inter-rater reliability $=80 \%$ ) who settled differences by discussion. No studies were excluded based on this quality assessment.

\section{Data synthesis and statistical analysis}

In case of sufficient homogeneity, meta-analyses and meta-regressions were to be conducted using metafor, a free package in $\mathrm{R}^{27}$

\section{RESULTS}

The systematic literature review resulted in 3404 identified hits (see figure 2). Twenty-one hits qualified for further

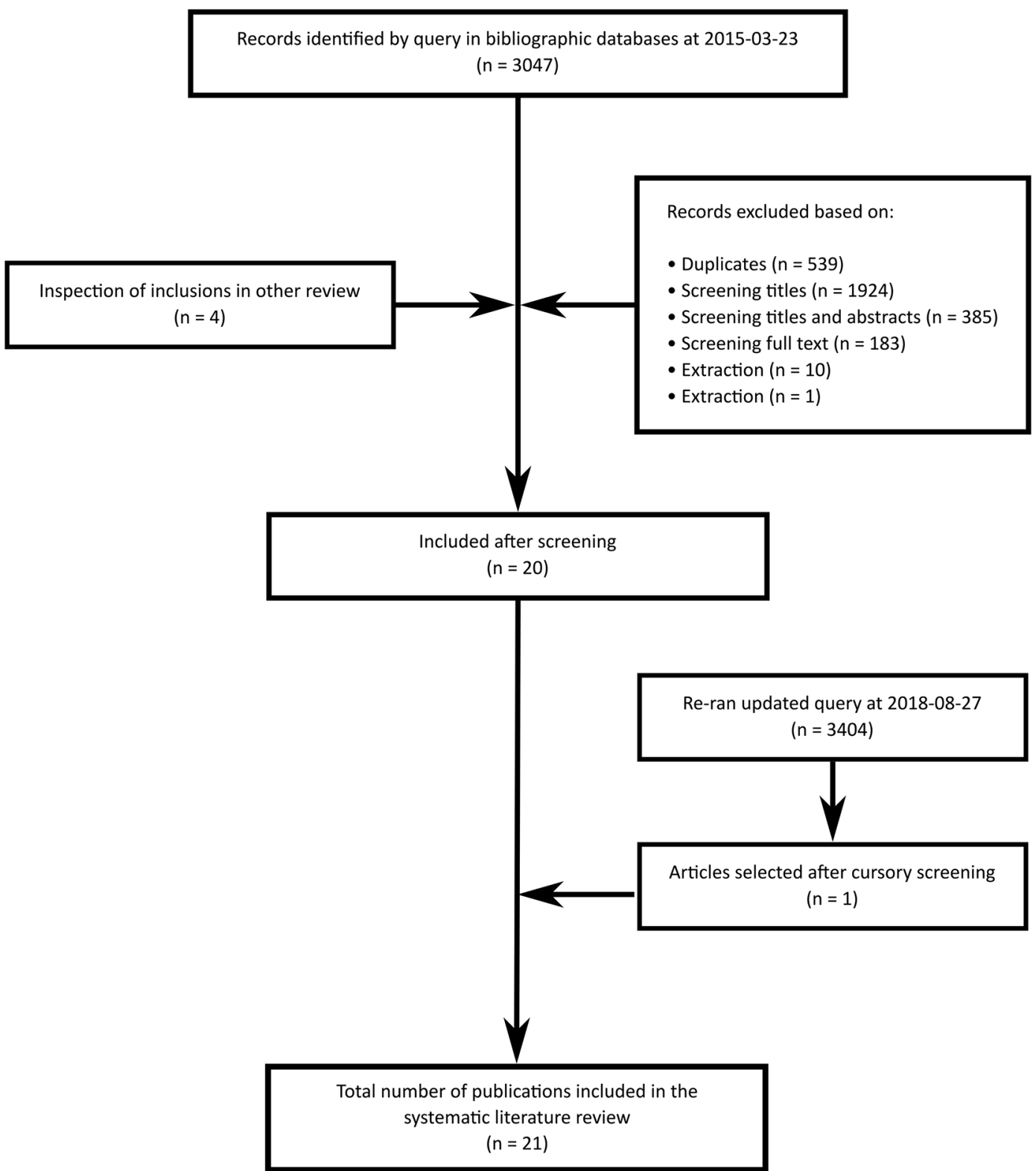

Figure 2 Flow chart of publications measuring maternal drinking behaviour(s) related to FASD included in the review. Details regarding the screening procedure and number of exclusions per exclusion criterion can be inspected at resource 2 . FASD, fetal alcohol spectrum disorders. 
screening and analysis. Hits were excluded because they were duplicates, not written in English or did not report associations between prenatal alcohol and FASD. The assessment of the included studies using the NOS revealed a wide range of quality scores with an average score of 6.57 out of 10 (for more details, see resource 5).

\section{Sample characteristics}

Sample characteristics can be inspected in table 1 . First, inspection of the data shows that the included studies were reported from five different countries, including Australia $(n=2)$, Croatia $(n=1)$, Italy $(n=2)$, South Africa $(n=12)$ and USA $(n=4)$. All studies were conducted after the year 1992. Almost all studies relied on interviews $(n=17)$, followed by self-reports $(n=3)$ and medical records $(n=1)$. Moreover, all studies were based on a retrospective sampling method. Behaviour was described in terms of maternal alcohol drinking related to a FASD diagnosis. Behaviours were reported before and during pregnancy where the period during pregnancy was specified per trimester (eg, first, second and third).

Further inspection shows that alcohol consumption was operationalised differently in each study (eg, dichotomous measures; a complete table can be found in table 1); in fact, no two studies used the same measure. Some studies reported units, whereas other studies reported subjective estimates (eg, many and less than). Others used dichotomous measures (eg, yes or no), a mixture of ordinal measures (eg, none, mild, moderate and heavy) or interval variables (eg, percentage). The original author's conclusions on maternal drinking behaviours and FASD can be inspected in table 2 .

\section{Dichotomous measures}

Dichotomous measures (eg, yes vs no) were available for 12 studies representing 44 measures (see table 1). These included questions concerning alcohol consumption before pregnancy. ${ }^{7} 101120$ Questions concerning alcohol consumption during pregnancy ${ }^{2} 814$ included the following variables: binge drinking without specifying how this was defined, ${ }^{2}{ }^{8}$ alcoholism, ${ }^{1}$ binge drinking (three or more drinks per occasion; five or more drinks per occasion), ${ }^{9}{ }^{10}{ }^{12-14}$ alcohol consumption in general, ${ }^{7911} 1719$ smoking as well as binge drinking (three or more drinks per occasion; five or more drinks per occasion. ${ }^{13}$ Moreover, questions were measured if pregnant women drank alcohol during the first trimester of pregnancy, ${ }^{791113141721}$ second trimester, ${ }^{791113141721}$ and/ or third trimester. ${ }^{791113141721}$ For more detailed information, see resource 5 .

\section{Nominal measures}

Although alcohol consumption is in fact a continuous variable, it was still operationalised at the nominal level in six nominal measures used in two studies. ${ }^{417}$ For more detailed information, see resource 5 .

\section{Ordinal measures}

In total, 24 ordinal measures were used in eight studies (see also the numbered studies in table 1). These incorporated questions concerning alcohol consumption before pregnancy, ${ }^{3}$ sometimes specified in categories of units, for example, grams a week, stopped during drinking or drank less than current use, ${ }^{356820}$ and alcohol consumption during pregnancy, ${ }^{1}$ including variables measuring the categories of alcohol intake in units of, for example, grams a week. ${ }^{3}{ }^{15}$ Moreover, questions were measured for each trimester of pregnancy: alcohol consumption during first trimester of pregnancy ${ }^{520}$ whereby variables were specified with categories, for example, drank less or drank more than current use, ${ }^{5820}$ alcohol consumption during second trimester ${ }^{56820}$ using the categories, for example, drank less or drank more than current $u^{5} e^{56820}$ and alcohol consumption during the third trimester $^{5} 682^{20}$ whereby variables were specified with categories, for example, drank less or drank more than current use. ${ }^{56820}$ For more detailed information, see resource 5 .

\section{Continuous measures}

Surprisingly, continuous measures were only available for six studies. In total, these studies employed 29 measures (see table 1). These included questions concerning alcohol consumption before pregnancy ${ }^{2} 8101218$ where variables were sometimes specified in number of drinks, for example, a day or week, ${ }^{81014}$ and during pregnancy, ${ }^{1014}$ where variables were sometimes specified in number of drinks, for example, during a drinking day, week and weekend. $^{211} 121418$ Moreover, number of alcoholic drinks or drinking days were measured during the first trimester of pregnancy, ${ }^{2} 101214$ sometimes specified in numbers a day or estimated $\mathrm{BAC}^{8}$; number of drinks or drinking days during second trimester, ${ }^{2} 101214$ sometimes specified in numbers a day or estimated $\mathrm{BAC}^{8}$; and/or number of drinks or drinking days during third trimester, ${ }^{2} 101214$ sometimes specified in numbers a day or estimated BAC. ${ }^{8}$

\section{Integration}

Categorical variables were based on different answer options and cut-off values, which precluded further aggregation or integration. Operationalisations on a continuous level of measurement also displayed substantial variation. Where possible, we attempted to transform these continuous measures of alcohol consumption into the same metric (eg, one standard drink defined in grams). However, even this was hindered by heterogeneity in reported standard sizes (sometimes not reported at all), types of alcohol described and other variation across countries. Moreover, few studies reported continuous data. Because of these reasons, conducting meta-analyses of the continuous variables alone was not feasible.

Consultation with three independent alcohol experts (eg, expertise in pharmacology of alcohol and measurements of alcohol drinking behaviours) revealed that 
Table 1 Overview of characteristics of included studies in this review

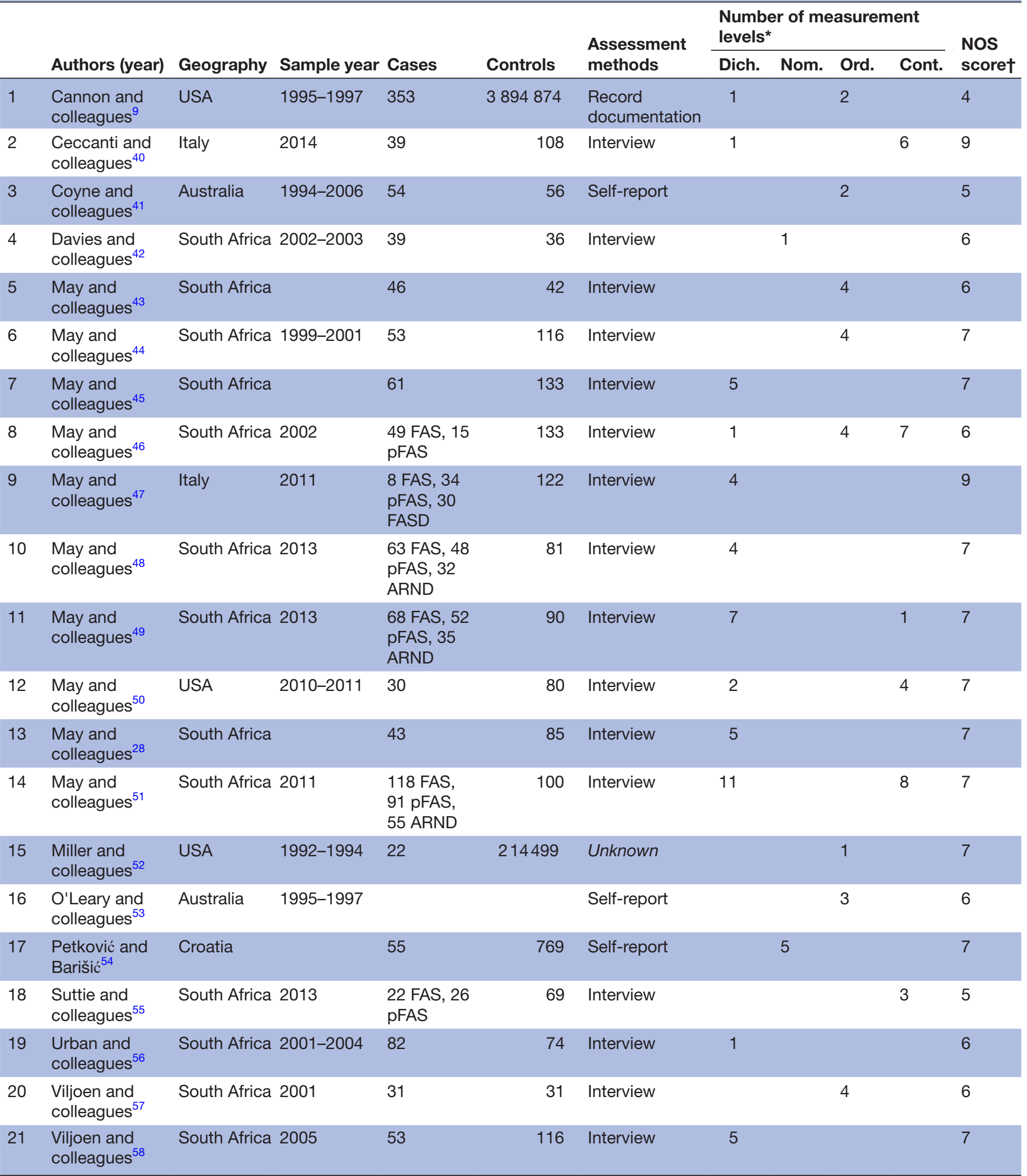

*Measurements of maternal alcohol drinking behaviour are categorised in three different levels: dichotomous ('Dich.', eg, yes/no), nominal ('Nom.', eg, admitted, negative and unanswered), ordinal ('Ord.', eg, <4 drinks and >4 drinks), continuous ('Cont.', eg, \%). The measures represent the different questions asked for each category (eg, 'drank during the first trimester of pregnancy'). †Each study was assessed using the adapted version of the Newcastle-Ottawa Scale (NOS). Scores were allocated from a scale from 0 (poor quality) to a maximum of 10 stars (excellent quality). For more detailed information, see resource 5.

ARND, alcohol-related neurodevelopmental disorder; FAS, fetal alcohol syndrome; FASD, fetal alcohol spectrum disorders; PFAS, partial fetal alcohol syndrome. 
Table 2 Conclusions made by authors of included studies on maternal drinking behaviours and FASD

\section{Authors (year) Original authors' conclusions}

Cannon and 'Mothers of children with FAS have severe substance abuse behaviors including daily drinking, binge colleagues $^{9} \quad$ drinking'.

Ceccanti and 'Mothers of children with a FASD reported more drinking three months prior to pregnancy, more current colleagues $^{40}$ drinking, and endorsed questionnaire items indicating that solitary drinking was more common'.

Coyne and colleagues $^{41}$

Davies and

colleagues $^{42}$ 'Mothers of children with FAS reported heavy alcohol intake during pregnancy'. 'Twenty five mothers with a FASD diagnosed child (69\%) reported drinking alcohol, on average, every week

May and colleagues $^{43}$ during their pregnancy'.

'Most drinking is binge drinking. Even though the current drinking quantities reported by both subjects and controls were not high in absolute standards, the most important interpretation of the data is the large differential between subjects and controls. There is no doubt, however, that these mothers drank sufficiently to produce verifiable cases of fetal alcohol syndrome as severe as we have seen anywhere in the United States'.

May and colleagues $^{44}$

'Alcohol consumption was much greater for case mothers than for control mothers in all comparisons. Control mothers were more likely to have been abstainers or Light drinkers compared with case mothers, who showed significantly heavier drinking patterns and reported drinking at the same level (53\%-55\%)\%) or higher during pregnancy (32\%-34\%)\%) compared with current drinking levels'.

May and 'Measures of drinking during the index pregnancies are significantly associated with low intelligence and colleagues $^{45}$ frequent behavioral problems in the children. Reported drinking during pregnancy (.59), drinks per day (.48), three drinks or more per occasion (.51), and five drinks or more per occasion (.45), correlate highly with total dysmorphology in the children'.

May and 'In most every variable of maternal alcohol use and abuse, a spectrum emerged based on the final diagnosis colleagues $^{46}$ of the child with FAS, PFAS, and control. Alcohol use was greatest in quantity, frequency, and duration among the mothers of FAS children, and generally next most severe among mothers of PFAS children, while lowest among controls'.

May and 'Mothers of children with FASD report heavy current drinking and drinking during the s2nd and 3rd trimesters colleagues $^{47}$ of the index pregnancy'.

May and 'Binge drinking of at least two days a week during all trimesters in this population may produce FAS or PFAS, colleagues $^{48} \quad$ while mothers of children with ARND and exposed children without an FASD are most likely to reduce their average and peak alcohol consumption in the later trimesters'.

May and 'Mean number of drinks per week and drinking 3 and 5 or more drinks per occasion during pregnancy both colleagues $^{49} \quad$ illustrate the significant difference between mothers of FASD children and those of normal children'.

May and 'Mothers of children who had a FASD reported more drinking 3 months before pregnancy, and heavy drinking colleagues $^{50}$ by the father of children who had FASD'.

May and 'With patterns of heavy episodic (binge) drinking being the most harmful to the fetus'. colleagues $^{28}$

May and colleagues $^{51}$

Miller and colleagues $^{52}$

O'Leary and colleagues $^{53}$

Petković and Barišić ${ }^{54}$

'Outcomes, both physical and cognitive/behavioral, are especially poor among children who were exposed to the highest quantity and frequency of drinking, especially drinks per drinking day and three or more drinks per occasion in both the case control comparisons and the correlation analysis'.

\section{'Mothers of FAS cases were more likely to drink alcohol during pregnancy'.}

'Heavy PAE in the first trimester was associated with a more than fourfold increased risk of ARBDs. This association was specific to PAE in the first trimester. The finding of twofold increased odds of ARBDs after moderate levels of PAE during late pregnancy is likely because many women also had heavy first trimester exposure and reduced their alcohol intake as pregnancy progressed'.

'Confirmed pregnancy alcohol consumption in the FAS/PFAS group was higher (18.2\%) to observed frequency in the whole sample of questioned mothers (11.5\%) and significantly higher when compared to non-FAS/PFAS mothers (10.4\%)'.

Suttie and 'No differences were found for prenatal alcohol exposure between the HE subgroup with FAS/PFAS affinity colleagues $^{55} \quad$ (nonsyndromal heavy exposed with FAS/PFAS-like face signature [HE1]) versus theHE subgroup with control affinity (nonsyndromal heavy exposed with more control-like face signature [HE2]) $(\mathrm{P}<0.10)$ '.

Urban and 'Maternal drinking during pregnancy was much more frequently reported in mothers of children with FAS/ colleagues $^{56} \quad$ PFAS than in controls'. 
Table 2 Continued

\begin{tabular}{ll}
\hline Authors (year) & Original authors' conclusions \\
\hline $\begin{array}{l}\text { Viljoen and } \\
\text { colleagues }\end{array}$ & $\begin{array}{l}\text { 'Mothers of children with FAS drank significantly heavier than controls, especially for continues drinking } \\
\text { heavily (and/or increasing) throughout pregnancy. Control mothers drank less and drinking levels declined } \\
\text { during pregnancy. Episodic drinking on weekends was modal for both groups with bingeing 5+drinks was } \\
\text { normative during } 2 \text { constructive days for FAS mothers'. }\end{array}$ \\
$\begin{array}{l}\text { Viljoen and } \\
\text { colleagues }^{58}\end{array}$ & $\begin{array}{l}\text { 'Mothers of children with FAS drink more than controls, drink rapidly and drink heavily in an episodic fashion. } \\
\text { Moreover, they do not quit or cut down during pregnancy'. }\end{array}$
\end{tabular}

FASD, fetal alcohol spectrum disorders.

aggregation of variables in the current dataset was not feasible. This substantial heterogeneity in operationalisations hindered further meta-analyses, and therefore the data will be described qualitatively below with emphasis on the used operationalisations and timing of exposure.

Because aggregation of the evidence was not possible, we instead sought to explore the heterogeneity exhibited by the included studies (note that all 230 extracted effect sizes are available in file 'effectsizes.csv' and an overview of the used operationalisations in 'Alcohol use variables.csv', both in resource 6). Given the small number of included studies, we decided to inspect visualisations of the associations between study characteristics. We plotted the quality of the studies (NOS scores), study year, measurement level of the alcohol consumption operationalisation, recruitment setting and data collection methods.

These visualisations revealed interesting patterns. The quality of studies (NOS score) seems to improve over the years. Data derived from clinical records were mainly based on ordinal measures. NOS score appeared higher for studies where maternal alcohol history was based on interviews. Finally, NOS scores appeared higher for samples recruited through active case ascertainment, especially in schools. We have included these visualisations in resource 6.

The wide range of variation in operationalisations provided a unique opportunity to compare them. Continuous measures provide detailed information about specific units (eg, oz, standard drink and BAC). If reported similarly across studies, these could be further meta-analysed. However, this requires reporting all information needed to convert the reported statistics into grams or millilitres of alcohol to enable integration with results from other countries. Other challenges appear to be present for logical, nominal and ordinal measures (eg, cut-off scores). Some studies reported categories, for example, binge drinking including three or more drinks per occasion versus five or more drinks per occasion ${ }^{28}$ and less than four drinks a day versus more than four drinks a day. ${ }^{9}$ None of the studies reported a description and considerations of why certain cut-off scores were chosen. Cut-off scores likely often followed recommendations by health promotion agencies or suggestions from earlier studies, but without explicit specification, this remains unclear. Perhaps the difficulty of establishing sensible cut-off values partly explains this, as doing so requires evidence syntheses to determine where exactly the effects of the relevant behaviour becomes qualitatively different. Such evidence (eg, meta-analyses of maternal alcohol consumption patterns) is not yet available. However, this should lead researchers to employ continuous operationalisations for now, rather than selecting (more or less arbitrary) cut-off scores.

\section{DISCUSSION}

In this systematic literature review, we aimed to summarise available data of studies that reported maternal alcohol drinking behaviours in relation to FASD. Data were available for 21 studies. The majority of these 21 studies were based on retrospective self-reports or interviews. A substantial heterogeneity in the applied measures for alcohol consumption was observed. Studies were based on continuous and categorical measures (dichotomous, nominal and ordinal). Continuous measures included blood alcohol content, percentages of drinking days, and alcohol consumption in grams or ounces. Categorical measures employed a variety of cut-offs to distinguish the different categories. This heterogeneity was so substantial that it precluded meta-analyses. Therefore, it was not possible to answer the original research question: the extant literature does not enable any conclusions as to the relationship between maternal alcohol consumption and the likelihood of infants developing FASD. Instead, however, a wealth of suggestions for future research was distilled from the literature.

The most striking finding was the variation in measurement instruments that were employed to assess maternal drinking behaviour. Each of the 21 included studies operationalised measures of alcohol consumption differently. The majority of studies used categorical measures. This is not desirable as these impose a discontinuous scale using cut-off scores. Because, as this review evidences, there exists insufficient evidence to derive whether alcohol consumption (as relating to FASD risk) should be considered as a continuous or discontinuous scale, and where the cut-offs should lie in the case of a discontinuous scale, such cut-off scores are necessarily arbitrary to a degree. In addition, categorising continuous data discards variance, thereby potentially obfuscating associations between variables. ${ }^{29-31}$ The variation in cut-off scores exhibited in the studies included in this review supports this assumption 
of arbitrariness and prohibits aggregation of the data collected in those studies. When studies did use continuous measures, studies often did not report how many grams of alcohol were in one standard drink. By making assumptions (eg, based on the standard drink size in the country of data collection), we were able to convert most standard drink-based measures into grams of alcohol, but this was not always feasible.

\section{Strengths and limitations}

One of the reasons for this heterogeneity may be that none of the included studies were conducted primarily to investigate the association between maternal drinking behaviour and FASD; although both variables were frequently measured and reported, most studies were designed to determine prevalence or FASD symptoms. It appears that few or no studies have been designed specifically to empirically establish how maternal alcohol consumption in humans is related to the likelihood of FASD. Given the comprehensive set up of this literature review, it is unlikely that such attempts have been overlooked. The search query was very extensive, rendering omission of relevant keywords unlikely. Screening was conducted in three screening rounds by two independent screeners, and all records flagged for inclusion by one screener were retained for closer inspection. In addition, the ascendency approach was applied. Given that reports of studies where these variables were secondary measures preclude conclusions about this relationship, it is as yet not possible to establish which recommendations can be empirically justified. In other words, even though in some target populations a total abstinence recommendation does not seem feasible. Available literature as yet offers no clear guidance that enables exploring a recommendation that could balance feasibility for the target population with dangers to health. Moreover, Mamluk $e t a l^{2}$ underlined the lack of data to make robust conclusions on the harmful effects of prenatal alcohol exposure and the unborn child. However, our inspection of the literature did yield a number of valuable recommendations for future research.

\section{Recommendations}

The original aim of this review was to provide a first step on the road to theory-based and evidence-based intervention development. We had hoped that after identifying the risk related to different behavioural patterns, we could provide guidelines for prevention workers working with different target populations (eg, alcohol-dependent pregnant women or teenage mothers). The next step could then be to map the determinants of those behaviours in those populations (ie, why individuals engage in the relevant undesirable and desirable behaviours) ${ }^{33}$ so that these can be targeted by behaviour change principles ${ }^{34}$ that are then integrated into prevention campaigns. ${ }^{35}$ However, it seems that the literature as yet has little guidance to offer. Because designing effective interventions first and foremost requires a thorough understanding of the target behaviour(s), it is therefore important that future research considers the limitations identified in this review so that in the future, a clearer picture may emerge.

The first recommendation is addressed specifically to epidemiological researchers and is based on the observation that the majority of studies assessed maternal drinking as part of a prevalence study. Because these studies form the largest part of the available data regarding associations between maternal alcohol consumption and FASD outcomes, it is important to pay close attention to the measurement of alcohol consumption, even in epidemiological studies with different primary aims.

Second, in general, researchers should anticipate the need to aggregate their measures of alcohol consumption with measures from other studies: in other words, conversion to consumption in metric units, such as grams of alcohol, in a specified time period, such as week or month, should be possible. If such conversion cannot be performed, the study cannot contribute to an accumulation of evidence. For example, many studies did not specify what exactly constituted a unit of alcohol (ie, one standard drink). This means that it was necessary to try and identify the definition of a unit of alcohol in the country where the data were collected in the period where the data were collected, but even then the obtained definition was unreliable as sometimes researchers conduct studies away from their home country yet use their home countries' unit definitions when reporting the results. Another example is that if timing of exposure was not specified, it is not clear whether the behaviour occurred during the first, second or third trimester (or was an aggregate of those periods).

This recommendation translates into a number of specific suggestions. Most of these are covered by following guidelines for the measurement of alcohol consumption, such as those specified by Dawson ${ }^{36}$ and Sobell and Sobell, ${ }^{37}$ but specifically, it is recommended that future studies assessing specific maternal drinking behaviours should report at least the following (see below for the recommended approach in each case):

i. How the sample was selected (eg, retrospective) and which method was used (eg, convenience sampling method).

ii. The maternal characteristics variables (eg, age, descent and educational level).

iii. Which method (or specific questions) was used to assess maternal alcohol consumption (eg, alcohol timeline follow back approach).

iv. The timing of exposure when assessing maternal alcohol consumption (eg, first trimester pregnancy).

v. The frequency of exposure when assessing maternal alcohol consumption (eg, number of exposure sessions per week or month).

vi. The amount of alcohol consumed per exposure session. ${ }^{36}$

vii. The sample size. 
viii. What was considered as one standard drink using International System of Units (ie, grams or millilitres of alcohol).

ix. If discontinuous (categorical) measures cannot be avoided, clear justification of the employed cut-offs.

The third recommendation refers to the complexity of exploring the association between maternal alcohol consumption and filial FASD. One cannot recruit children with FASD and then proceed to select children without FASD. This is not helpful because the number of children without FASD but with parents with matched alcohol consumption patterns is the variable of interest. The proportion of children with FASD within each group of parents with a given alcohol consumption pattern is the dependent variable to measure. For example, let us assume that in the left panel of figure 1 (showing the sigmoid relationship), the probability of FASD is $1 \%$ if alcohol consumption is lower than five units; $25 \%$ if alcohol consumption is between 5 units and 10 units; $75 \%$ if alcohol consumption is between 10 units and 15 units; and $99 \%$ if alcohol consumption exceeds 15 units. Similarly, let us assume that in the right panel (showing the linear relationship), the probability of FASD is $12.5 \%$ is alcohol consumption is lower than 5 units; $37.5 \%$ is alcohol consumption is between 5 units and 10 units; $62.5 \%$ is alcohol consumption is between 10 units and 15 units; and $87.5 \%$ is alcohol consumption exceeds 15 units. This means that for 1000 parents consuming between 0 units and 5 units (the yellow area), in the sigmoid scenario, 10 children will develop FASD and 990 (99 times more) will not, while in the linear scenario, 125 children develop FASD and 875 will not (seven times more). Now, imagine that a researcher visits a school and screens all children for FASD, and 10 children screen positive for FASD. For simplicity's sake, let us assume that the parents of all these children happened to consume less than 5 units per week during pregnancy. Now, this researcher will not know whether to create a matched control group that is 99 times larger (as would be the case in the sigmoid scenario) or seven times larger (as would be the case in the linear scenario). It is exactly the relative sizes of these groups that is the variable to measure, and the only way to do so is to measure both maternal alcohol consumption patterns and filial FASD in a large sample.

Based on these recommendations, the ideal design would be a large-scale (Note that what constitutes 'largescale' depends on the expected FASD prevalence in a population as well as the target behaviour under investigation, eg, abstinence vs moderated drinking, or abstinence vs regular drinking patterns. These two parameters determine the effect size of the association that is to be estimated, which in turn enables computation of the required sample size for accurate estimation of that effect size using Accuracy in Parameter Estimation methods.) prospective study where maternal and paternal alcohol consumption patterns would be assessed both using self-reports (conform the recommendations made earlier) as well as objective measures such as biomarkers for alcohol consumption. ${ }^{38}$ Infants would then be assessed for FASD according to the revised IOM guidelines ${ }^{1}$ and other recommendations provided by Roozen et $a l^{6}{ }^{6}$ and the FASD prevalence would be related to alcohol consumption patterns of both parents separate and in conjunction. This design also enables examination of potential confounders such as social economic status or age. Such an ideal design may not always be feasible. After all, learning about the association of parental drinking patterns to filial FASD requires assessing drinking patterns in all pregnancies: it is not possible to start from identified FASD cases, as we explained earlier. However, even when other designs are used, it is important that researchers anticipate data aggregation over studies and therefore attempt to provide alcohol measures in metric units.

The present review focused on reported data on maternal drinking behaviours. Some of the included studies also reported paternal drinking patterns or grandparental drinking patterns. The role of paternal drinking and transgenerational toxicity on fetal development and FASD is not well understood. A recent review study by Gupta and colleagues ${ }^{19}$ reported that paternal alcoholism alters the gene expression for fetal susceptibility to FAS. In another review, Resendiz and colleagues ${ }^{20}$ argue that transgenerational toxicity may play a role in FASD aetiology. Moreover, social facilitation by paternal drinking is significantly associated with maternal drinking. ${ }^{39}$ The origin of FASD is therefore based on maternal drinking behaviours and by many other factors (eg, genetic and epigenetic predisposition, maternal body makeup and lifestyle). Gupta and colleagues ${ }^{19}$ emphasised that FAS aetiology, and also other diagnosis within the FASD spectrum, is based on a complex interaction of different factors whereby cautious interpretation is warranted.

\section{CONCLUSION}

The current knowledge on maternal alcohol drinking behaviours in relation to FASD is limited. Behaviours were measured using various techniques and operationalised differently. For evidence-based preventive measures, it is necessary to identify which prenatal alcohol drinking behaviour(s) are most in need of intervention. Several recommendations have been made that can facilitate accumulation of evidence over studies. Following these recommendations can contribute to establishing the evidence base required for the development of effective preventive health promoting programmes.

Author affiliations

${ }^{1}$ Governor Kremers Centre, Maastricht University Medical Centre, Maastricht, The Netherlands

${ }^{2}$ Department of Work and Social Psychology, Maastricht University, Maastricht, The Netherlands

${ }^{3}$ Faculty of Psychology and Education Science, Open University of The Netherlands, Heerlen, The Netherlands

${ }^{4}$ Department of Health, Ethics \& Society, Maastricht University, Maastricht, The Netherlands 
${ }^{5}$ Department of Obstretrics and Gynaecology, Maastricht University Medical Centre, Maastricht, The Netherlands

${ }^{6}$ Division of Gastroenterology and Hepatology, Department of Internal Medicine, Maastricht University Medical Centre, Maastricht, The Netherlands

Contributors SR, G-JYP, GerjK and LC designed the study and directed its implementation, including quality assurance and control. Authors DT, JN and GerK helped supervising the field activities. SR and G-JYP conducted the literature review and analyses and prepared the Materials and Methods and the Discussion sections of the text. All other coauthors contributed to successive drafts. All authors gave significant input in preparation of the article and approved the manuscript and submission.

Funding The authors have not declared a specific grant for this research from any funding agency in the public, commercial or not-for-profit sectors.

Competing interests None declared.

Patient consent for publication Not required.

Provenance and peer review Not commissioned; externally peer reviewed.

Data sharing statement All data, analysis scripts and other materials are publicly available at the Open Science Framework repository for this study (https://osf.io/ whq45/).

Open access This is an open access article distributed in accordance with the Creative Commons Attribution Non Commercial (CC BY-NC 4.0) license, which permits others to distribute, remix, adapt, build upon this work non-commercially, and license their derivative works on different terms, provided the original work is properly cited, appropriate credit is given, any changes made indicated, and the use is non-commercial. See: http://creativecommons.org/licenses/by-nc/4.0/.

\section{REFERENCES}

1. Hoyme HE, Kalberg WO, Elliott AJ, et al. Updated clinical guidelines for diagnosing fetal alcohol spectrum disorders. Pediatrics 2016;138:e20154256.

2. American Psychiatric Association. Diagnostic and statistical manual of mental disorders (DSM-5). Washington DC: American Psychiatric Association, 2013.

3. BMA Board of Science. Fetal alcohol spectrum disorders A guide for healthcare professionals: British Medical Association, 2007.

4. May PA, Gossage JP, Kalberg WO, et al. Prevalence and epidemiologic characteristics of FASD from various research methods with an emphasis on recent in-school studies. Dev Disabil Res Rev 2009;15:176-92.

5. Popova S, Lange S, Probst C, et al. Estimation of national, regional, and global prevalence of alcohol use during pregnancy and fetal alcohol syndrome: a systematic review and meta-analysis. Lancet Glob Health 2017;5:e290-e299.

6. Roozen S, Peters GJ, Kok G, et al. Worldwide prevalence of fetal alcohol spectrum disorders: a systematic literature review including meta-analysis. Alcohol Clin Exp Res 2016;40:18-32.

7. Esper LH, Furtado EF. Identifying maternal risk factors associated with Fetal Alcohol Spectrum Disorders: a systematic review. Eur Child Adolesc Psychiatry 2014;23:877-89.

8. May PA, Tabachnick BG, Gossage JP, et al. Maternal risk factors predicting child physical characteristics and dysmorphology in fetal alcohol syndrome and partial fetal alcohol syndrome. Drug Alcohol Depend 2011:119:18-27.

9. Cannon MJ, Dominique Y, O'Leary LA, et al. Characteristics and behaviors of mothers who have a child with fetal alcohol syndrome. Neurotoxicol Teratol 2012;34:90-5.

10. Henderson J, Gray R, Brocklehurst P. Systematic review of effects of low-moderate prenatal alcohol exposure on pregnancy outcome. BJOG: An International Journal of Obstetrics \& Gynaecology 2007;114:243-52.

11. Flak AL, Su S, Bertrand J, et al. The association of mild, moderate, and binge prenatal alcohol exposure and child neuropsychological outcomes: a meta-analysis. Alcohol Clin Exp Res 2014;38:214-26.

12. Davis PJ, Partridge JW, Storrs CN. Alcohol consumption in pregnancy. How much is safe? Arch Dis Child 1982;57:940-3.

13. BMA Board of Science. Alcohol and pregnancy preventing and managing fetal alcohol spectrum disorders, 2016.

14. Sullivan EV, Pfefferbaum A. Fetal alcohol spectrum disorder: pathogenesis and mechanisms. Alcohol Nerv Syst Handb Clin Neurol $2014 ; 125$.
15. Ramsay M. Genetic and epigenetic insights into fetal alcohol spectrum disorders. Genome Med 2010;2:27.

16. Ehrhart F, Roozen S, Verbeek J, et al. Review and gap analysis: molecular pathways leading to fetal alcohol spectrum disorders. $\mathrm{Mol}$ Psychiatry 2018

17. Jonsson E, Salmon A, Warren KR. The international charter on prevention of fetal alcohol spectrum disorder. Lancet Glob Health 2014;2:e135-e137.

18. WHO. Guidelines for the identification and management of substance use and substance use disorders in pregnancy. Geneva 2014.

19. Gupta KK, Gupta VK, Shirasaka T. An update on fetal alcohol syndrome-pathogenesis, risks, and treatment. Alcohol Clin Exp Res 2016;40:1594-602

20. Resendiz M, Chen Y, Oztürk NC, et al. Epigenetic medicine and fetal alcohol spectrum disorders. Epigenomics 2013;5:73-86.

21. Kok G, Gottlieb NH, Peters GJ, et al. A taxonomy of behaviour change methods: an Intervention Mapping approach. Health Psychol Rev 2016;10:297-312.

22. May PA, Gossage JP. Maternal risk factors for fetal alcohol spectrum disorders: not as simple as it might seem. Alcohol Res Heal $2011 ; 34: 15-26$.

23. Strecher VJ, Seijts GH, Kok GJ, et al. Goal setting as a strategy for health behavior change. Health Educ Q 1995;22:190-200.

24. Moher D, Liberati A, Tetzlaff J, et al. Preferred reporting items for systematic reviews and meta-analyses: the PRISMA statement. Ann Intern Med 2009;151:264-9.

25. R Development Core Team. R: A Language and Environment for Statistical Computing, 2014.

26. Wells GA, Shea B, O'Connell D, et al. The Newcastle-Ottawa Scale (NOS) for assessing the quality of nonrandomized studies in metaanalyses, 2013.

27. Viechtbauer W. Conducting meta-analyses in $R$ with the metafor Package. J Stat Softw 2010;36:1-48.

28. May PA, Hamrick KJ, Corbin KD, et al. Dietary intake, nutrition, and fetal alcohol spectrum disorders in the Western Cape Province of South Africa. Reprod Toxicol 2014;46:31-9.

29. DeCoster J, Iselin AM, Gallucci M. A conceptual and empirical examination of justifications for dichotomization. Psychol Methods 2009;14:349-66.

30. MacCallum RC, Zhang S, Preacher KJ, et al. On the practice of dichotomization of quantitative variables. Psychol Methods 2002;7:19-40.

31. Altman DG, Royston P. The cost of dichotomising continuous variables. BMJ 2006;332:1080.1.

32. Mamluk L, Edwards HB, Savović J, et al. Low alcohol consumption and pregnancy and childhood outcomes: time to change guidelines indicating apparently 'safe' levels of alcohol during pregnancy? A systematic review and meta-analyses. BMJ Open 2017;7:e015410.

33. Peters G-JY, Crutzen R. Establishing determinant importance using CIBER: an introduction and tutorial. Eur Heal Psychol 2018;20.

34. Crutzen R, Peters GY. Evolutionary learning processes as the foundation for behaviour change. Health Psychol Rev 2018;12:43-57.

35. Roozen S, Black D, Peters GY, et al. Fetal Alcohol Spectrum Disorders (FASD): an approach to effective prevention. Curr Dev Disord Rep 2016;3:229-34.

36. Dawson DA. Methodological issues in measuring alcohol use. Alcohol Res Health 2003;27:18-29.

37. Sobell LC, Sobell MB. Alcohol consumption measures. Assessing alcohol problems: a guide for clinicians and researchers, 1995:75-99.

38. Chabenne A, Moon C, Ojo C, et al. Biomarkers in fetal alcoho syndrome. Biomarkers and Genomic Medicine 2014;6:12-22.

39. McBride N, Johnson S. Fathers' role in alcohol-exposed pregnancies. systematic review of human studies. Am J Prev Med 2016;9.

40. Ceccanti M, Fiorentino D, Coriale G, et al. Maternal risk factors for fetal alcohol spectrum disorders in a province in Italy. Drug Alcohol Depend 2014;145:201-8.

41. Coyne KL, de Costa CM, Heazlewood RJ, et al. Pregnancy characteristics of women giving birth to children with fetal alcohol syndrome in Far North Queensland. Aust N Z J Obstet Gynaecol 2008;48:240-7.

42. Davies L, Dunn M, Chersich M, et al. Developmental delay of infants and young children with and without fetal alcohol spectrum disorder in the Northern Cape Province, South Africa. Afr J Psychiatry 2011;14:298-305.

43. May PA, Brooke L, Gossage JP, et al. Epidemiology of fetal alcohol syndrome in a South African community in the Western Cape Province. Am J Public Health 2000;90:1905-12.

44. May PA, Gossage JP, Brooke LE, et al. Maternal risk factors for fetal alcohol syndrome in the Western cape province of South Africa: a population-based study. Am J Public Health 2005;95:1190-9. 
45. May PA, Gossage JP, Marais AS, et al. The epidemiology of fetal alcohol syndrome and partial FAS in a South African community. Drug Alcohol Depend 2007;88:259-71.

46. May PA, Gossage JP, Marais AS, et al. Maternal risk factors for fetal alcohol syndrome and partial fetal alcohol syndrome in South Africa: a third study. Alcohol Clin Exp Res 2008;32:738-53.

47. May PA, Fiorentino D, Coriale G, et al. Prevalence of children with severe fetal alcohol spectrum disorders in communities near Rome, Italy: new estimated rates are higher than previous estimates. Int J Environ Res Public Health 2011;8:2331-51.

48. May PA, Blankenship J, Marais AS, et al. Maternal alcohol consumption producing fetal alcohol spectrum disorders (FASD): quantity, frequency, and timing of drinking. Drug Alcohol Depend 2013;133:502-12.

49. May PA, Blankenship J, Marais AS, et al. Approaching the prevalence of the full spectrum of fetal alcohol spectrum disorders in a South African population-based study. Alcohol Clin Exp Res 2013;37:818-30.

50. May PA, Baete A, Russo J, et al. Prevalence and characteristics of fetal alcohol spectrum disorders. Pediatrics 2014;134:855-66.

51. May P, De Vries M, Marais A-S, et al. Replication of high fetal alcohol spectrum disorders prevalence rates, child characteristics, and maternal risk factors in a second sample of rural communities in South Africa. Int J Environ Res Public Health 2017;14:522.

52. Miller LA, Shaikh T, Stanton C, et al. Surveillance for fetal alcohol syndrome in Colorado. Public Heal Rep 1995;110:690-7.

53. O'Leary CM, Nassar N, Kurinczuk JJ, et al. Prenatal alcohol exposure and risk of birth defects. Pediatrics 2010;126:e843-e850.

54. Petković G, Barišić I. Prevalence of fetal alcohol syndrome and maternal characteristics in a sample of schoolchildren from a rural province of Croatia. Int J Environ Res Public Health 2013;10:1547-61.

55. Suttie M, Foroud T, Wetherill L, et al. Facial dysmorphism across the fetal alcohol spectrum. Pediatrics 2013;131:788-.

56. Urban M, Chersich MF, Fourie LA, et al. Fetal alcohol syndrome among grade 1 schoolchildren in Northern Cape Province: prevalence and risk factors. S Afr Med J 2008;98:877-82.

57. Viljoen D, Croxford J, Gossage JP, et al. Characteristics of mothers of children with fetal alcohol syndrome in the Western Cape Province of South Africa: a case control study. J Stud Alcohol 2002;63:6-17.

58. Viljoen DL, Gossage JP, Brooke L, et al. Fetal alcohol syndrome epidemiology in a South African community: a second study of a very high prevalence area. J Stud Alcohol 2005;66:593-604. 